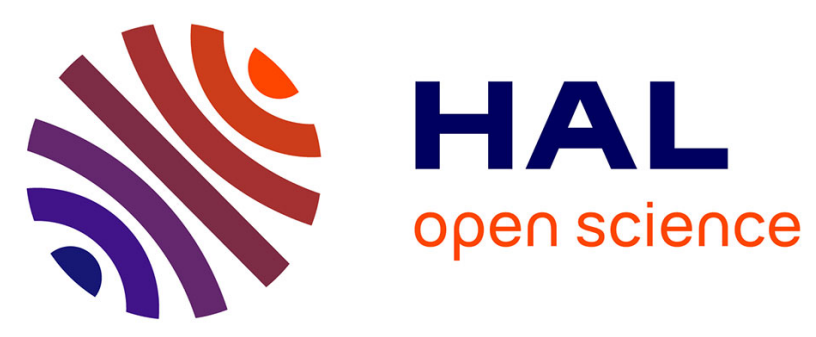

\title{
Sporopollenin exine capsules (SpECs) derived from Lycopodium clavatum provide practical antioxidant properties by retarding rancidification of an omega-3 oil
}

M.J. Thomasson, A. Diego-Taboada, S. Barrier, J. Martin-Guyout, E. Amedjou, S.L. Atkin, Yves Queneau, A.N. Boa, G. Mackenzie

\section{To cite this version:}

M.J. Thomasson, A. Diego-Taboada, S. Barrier, J. Martin-Guyout, E. Amedjou, et al.. Sporopollenin exine capsules (SpECs) derived from Lycopodium clavatum provide practical antioxidant properties by retarding rancidification of an omega-3 oil. Industrial Crops and Products, 2021, 154, pp.112714. 10.1016/j.indcrop.2020.112714 . hal-02887257

\section{HAL Id: hal-02887257 \\ https://hal-udl.archives-ouvertes.fr/hal-02887257}

Submitted on 8 Jul 2020

HAL is a multi-disciplinary open access archive for the deposit and dissemination of scientific research documents, whether they are published or not. The documents may come from teaching and research institutions in France or abroad, or from public or private research centers.
L'archive ouverte pluridisciplinaire HAL, est destinée au dépôt et à la diffusion de documents scientifiques de niveau recherche, publiés ou non, émanant des établissements d'enseignement et de recherche français ou étrangers, des laboratoires publics ou privés. 


\title{
Sporopollenin exine capsules (SpECs) derived from Lycopodium clavatum provide practical antioxidant properties by retarding rancidification of an $\omega-3$ oil
}

\author{
Matthew J. Thomasson a, Alberto Diego-Taboada a, b, Sylvain Barrier a, Jules Martin-Guyout a, \\ Elizabeth Amedjou ${ }^{a}$, Stephen L. Atkin ${ }^{\text {b, c }}$, Yves Queneau ${ }^{\text {a, d }}$, Andrew N. Boa ${ }^{\mathrm{a}}{ }_{*}$, Grahame Mackenzie ${ }^{\mathrm{a}, \mathrm{b}}{ }_{*}$ \\ a Department of Chemistry \& Biochemistry, University of Hull, Hull, HU6 7RX, UK \\ ${ }^{\mathrm{b}}$ Sporomex Ltd., Medina House, 2 Station Avenue, Bridlington, YO16 4LZ, UK \\ ${ }^{c}$ Royal College of Surgeons in Ireland - Medical University of Bahrain, Al Sayh Muharraq Governorate, Bahrain \\ ${ }^{\mathrm{d}}$ Univ Lyon, Université Claude Bernard Lyon 1, CNRS, INSA Lyon, CPE Lyon, ICBMS, UMR 5246, Bât. E. Lederer, 1 rue Victor Grignard, F-69622 Villeurbanne, France
}

\section{A R T ICLE INFO}

\section{Keywords:}

Sporopollenin

Exine

Microcapsule

Omega-3 oil

Antioxidant

Peroxide value

\begin{abstract}
A B S T R A C T
In recent years the use of natural antioxidants in foodstuffs and personal care products has become increasingly important for consumers and therefore manufacturers. In this work, sporopollenin exine capsules (SpECs), extracted from spores of the common club moss Lycopodium clavatum L, have been shown to protect an $\omega-3$ oil from oxidation caused by natural light or accelerated oxidation with UV irradiation. The mechanism of action has been shown to be principally by free radical quenching as opposed to light shielding, supported by evidence of similarity in levels of protection when the ratio of SpECs to oil was $0.2 \% \mathrm{w} / \mathrm{v}$ compared with $50 \% \mathrm{w} / \mathrm{v}$. The antioxidant effect is not materially altered by the extraction process from the raw material and is clearly an inherent property of the sporopollenin contained in the spores of $L$. clavatum due to the accessible phenolic groups on the surface on the SpECs. These results provide promising evidence that SpECs could be useful as a bio-sourced antioxidant for protecting $\omega-3$ oils and related oxidation-prone molecules.
\end{abstract}

\section{Introduction}

Rancidification is a process by which full or partial oxidation of unsaturated oils leads to the formation of small molecule ketones and aldehydes. Often associated with oil-containing foods, rancidification results in unpleasant flavours and odours (Allen and Hamilton, 1994), often to the degree that the foods are rendered inedible and thrown away. Therefore, it is common practice to add an antioxidant to protect the oils and fats from such processes, and thus enhance the shelf life of a product. There are a wide range of other commercial products that are also sensitive to oxidative processes. For example, nutraceuticals (e.g. ubiquinol, eicosapentaenoic acid, and docosahexaenoic acid) may be degraded and rendered inactive, and industrially produced vinyl monomers, which are naturally prone to radical reactions, can polymerise during storage or shipping.

It is known that bee pollen (Leja et al., 2007) and the spores of L. clavatum L. (club moss) (Orhan et al., 2007) possess antioxidant activity. A key component of these grains is the outer protective layer, known as the exine, which is made of the natural biopolymer sporopollenin. This biopolymer contains covalently bound conjugated phenols such as ferulic, coumaric (Wehling et al., 1989; Li et al., 2019), and cinnamic acids (Rozema et al., 2001a; Herminghaus et al., 1988; Schulze Osthoff and Wiermann, 1987), all of which are known individually to possess antioxidant activity (Prior et al., 2005; Meuter-Gerhards et al., 1995). It is relevant to note here that a recent and contradictory report suggests that aromatic components are not present in sporopollenin, but the report does suggest it contains hydroxypyrone moieties (Mikhael et al., 2020). It seems reasonable to suggest that the presence of such a range of functional groups, either covalently attached to or part of the exine skeleton, is a result of an evolutionary process to help protect the nutrients and genetic components of pollen or spore grains against UV damage and oxidation. However, based on work by Rozema and co-workers (Rozema et al., 2001b), the capacity of the exine to shield fully against UV light is apparently incomplete. They determined values of the relative UV $-\mathrm{B}$ absorbance, integrated over the wavelength range $280-320 \mathrm{~nm}$, for suspensions of sporopol-

\footnotetext{
* Corresponding authors at: Department of Chemistry \& Biochemistry, University of Hull, Hull, HU6 7RX, UK.

Email address: a.n.boa@hull.ac.uk (A.N. Boa)
} 
lenin in glycerol and proposed that "the pollen wall is effective in screening out more than $80 \%$ of the incident UV radiation." A more recent study (Atkin et al., 2011), involving isolated sporopollenin exine capsules (SpECs) from different species, showed that the light transmission of a single exine capsule wall is approximately $50 \%$, due mainly to light absorption. Interestingly, this value was similar over the light wavelength range $200-900 \mathrm{~nm}$ for the three different species of plants studied. Therefore, it would seem reasonable that the conjugated groups in sporopollenin, resulting from evolution, have a key role in acting as a photochemical shield. Furthermore, there is a wealth of evidence showing sporopollenin exines possess distinct physical robustness and chemical stability (Mackenzie et al., 2015). The exine layer thus appears to have a key, multifunctional role in protecting the internal cytoplasmic materials of the pollen or spore grain against sunlight and air, and in transit by wind or insect to the site of pollination. Unlike raw pollen, which hay fever sufferers will attest to triggering seasonal allergies, SpECs extracted by base or acid catalysed hydrolyses, have been shown to be protein free (Diego-Taboada et al., 2013) and non-allergenic. Therefore, it was surmised that SpECs might prove to be a bioinspired and natural alternative to small, synthetic molecules for the protection of unsaturated materials towards oxidation.

Other particulate antioxidants containing natural phenolic moieties have been reported in the literature. Bai and co-workers prepared ethyl $\alpha$-linolenate emulsions using caffeic acid-grafted chitosan as droplet stabilisers (Bai et al., 2019). The droplets were then freeze dried to give particles of uniform size, and it was found that the caffeic acid component of the 'wall material' acted as an antioxidant to protect the fatty acid contained within. Torkamani and co-workers made gelatin nanospheres to encapsulate phenolic antioxidant-containing plant extracts from Momordica charantia (Torkamani et al., 2018). Narignin is a hydroxyflavanone-glycoside found in citrus fruits. Sahiner and co-workers prepared and characterized biodegradable antioxidant poly(naringin) particles in the hundreds of nanometer to lower micrometer size range (Sahiner et al., 2018). A much simpler approach to the production of microparticulate antioxidants simply involves grinding dried plant materials. Soualeh and co-workers investigated the role of particle size on the antioxidant and cytoprotective activity of powdered plant material (Soualeh et al., 2018). Smaller (50-100 $\mu \mathrm{m}$ ) particles proved to be more effective than medium $(100-180 \mu \mathrm{m})$ or larger particles $(180-315 \mu \mathrm{m})$ at protecting against hydrogen peroxide-induced oxidative stress of mouse spleen cells. A similar relationship between particle size and antioxidant ability was observed by both Nabil and co-workers, and Zaiter and co-workers, who investigated powders from Opuntia ficus-indica and catechin-containing green tea respectively (Nabil et al., 2019; Zaiter et al., 2016). The particulate antioxidants described above fall into two broad categories; the powdered plant materials provide particles quickly but contain a complex mixture of compounds, whereas the synthetic particles are more complex, artificial constructs but containing a simpler number of components. In comparison, the SpECs in this work have a more regular composition, yet are extracted in usable form via a simple process directly from the bio-resource.

Previously, SpECs have been used to encapsulate $\omega-3$ oils, and interestingly this encapsulant was found to mask the oil's taste (Barrier et al., 2010). Antioxidant effects of isolated L. clavatum SpECs have also been observed (Atkin et al., 2007), and the redox activity of SpECs investigated electrochemically (Thomasson et al., 2010). These results were consistent with the presence of conjugated phenolics as a constituent part of the exine shell of the capsules. Boehm-type titrations with isolated $L$. clavatum SpECs estimated the total phenolic content of the sporopollenin to be in the order of $0.35 \mathrm{mmol} / \mathrm{g}$ (Diego-Taboada et al., 2012). Subsequently, Smirnova and co-workers (Smirnova et al., 2012) examined the redox properties of the sporopollenin from Nico- tiana tabacum L. using EPR, but noted that treatment of the sporopollenin capsules with sodium hydroxide diminished their redox properties. This was attributed to hydrolysis and loss of conjugated phenolic esters. There is as yet no literature showing the practical antioxidant properties of SpECs with regard to protecting a sensitive material and over an extended time frame. Therefore, this study set out to demonstrate the capacity of SpECs to protect an unsaturated fatty acid against oxidation in a practical setting, with a view to wider investigations of their use to protect related, oxidatively-sensitive compounds.

\section{Materials and methods}

L. clavatum spores were purchased from Tibrewala International (Nepal), 2,2-Diphenyl-1-picrylhydrazyl (DPPH) was purchased from Sigma Life Science, and all other chemicals and reagents were purchased from either Sigma-Aldrich or Fisher Scientific UK Ltd and used as received. An oxidation-sensitive $\omega-3$ oil (Seven Seas ${ }^{\circledR}$ 'Simply Timeless ${ }^{\circledR}$ ' Omega-3 cod liver oil Plus) was purchased from local high street retailers. When not in use, the $\omega-3$ oil was stored tightly sealed in its original bottle, covered in aluminium foil, and in the refrigerator $\left(5-10^{\circ} \mathrm{C}\right)$, but was allowed to warm to room temperature unopened before use. For peroxide value (PV) titrations, fresh starch indicator solution was prepared daily. After a test titration, to determine the approximate endpoint, subsequent accurate titrations were conducted in which the indicator was added a few millilitres short of the expected endpoint. At the start of each day's experiments, the $\omega-3$ oil was titrated to check that the PV was below 10 (typically 4-7). If it was above 9 then the oil was discarded, and a fresh, unopened bottle started. Where filtration of SpECs from a suspension was involved, a grade 3 porosity sintered funnel was used.

\subsection{Extraction of sporopollenin exine capsules (SpECs) from $L$. clavatum spores}

The extraction of SpECs using a phosphoric acid step was carried out as described previously (Atkin et al., 2005), with slight modifications. Other abbreviated extraction protocols only used either hydrochloric acid or sodium hydroxide alone. After the careful washing and drying stages described below, all methods produced free-flowing powders, which were typically dark beige in colour.

\subsubsection{Phosphoric acid hydrolysed spores (PHS)}

Raw L. clavatum spores $(200 \mathrm{~g})$ were suspended in acetone $\left(900 \mathrm{~cm}^{3}\right)$ and heated under reflux for $4 \mathrm{~h}$. The spores were recovered by filtration, washed with acetone $\left(250 \mathrm{~cm}^{3}\right)$ and air dried overnight. The resulting solid was suspended in potassium hydroxide $\left(6 \% \mathrm{w} / \mathrm{v} ; 900 \mathrm{~cm}^{3}\right)$, stirred at $80^{\circ} \mathrm{C}$ for $6 \mathrm{~h}$, recovered by filtration, and washed with hot water $\left(2 \times 500 \mathrm{~cm}^{3}\right)$. The solids were then re-suspended in fresh potassium hydroxide, heated at $80^{\circ} \mathrm{C}$ for a further $6 \mathrm{~h}$, and again recovered by filtration before washing with hot water $\left(6 \times 500 \mathrm{~cm}^{3}\right)$. The solids were suspended in ortho-phosphoric acid $\left(85 \% ; 800 \mathrm{~cm}^{3}\right)$ and stirred at $60^{\circ} \mathrm{C}$ for 5 days. The product was recovered by filtration, washed with hot water $\left(2 \times 500 \mathrm{~cm}^{3}\right), 2 \mathrm{M}$ sodium hydroxide $\left(2 \times 500 \mathrm{~cm}^{3}\right)$, hot water $\left(6 \times 500 \mathrm{~cm}^{3}\right)$, phosphate buffered saline (PBS; $\left.2 \times 250 \mathrm{~cm}^{3}\right)$ and finally hot water $\left(2 \times 500 \mathrm{~cm}^{3}\right)$. The product was then suspended in ethanol $\left(900 \mathrm{~cm}^{3}\right)$ and stirred at $80^{\circ} \mathrm{C}$ for $4 \mathrm{~h}$. The SpECs were recovered by filtration, washed with ethanol $\left(500 \mathrm{~cm}^{3}\right)$, suspended in acetone and sonicated for $30 \mathrm{~min}$ before being recovered by filtration, washed with acetone $\left(500 \mathrm{~cm}^{3}\right)$, and dried under vacuum before transferring to an oven at $60^{\circ} \mathrm{C}$ for further drying. Combustion elemental analysis found: C, $62.59 \pm 0.88 \% ; \mathrm{H}, 8.02 \pm 0.44 \% ; \mathrm{N}, 0.00 \pm 0.00 \%(N=4)$. 


\subsubsection{Base hydrolysed spores (BHS)}

2.1.2.1. Method 1- a longer method providing BHS1 SpECs Base hydrolysed spores (BHS) were prepared following the protocol described above for phosphoric acid hydrolysed spores (Section 2.1.1), but with omission of the extended heating with phosphoric acid. Combustion elemental analysis found: C, $61.65 \pm 0.61 \% ; \mathrm{H}, 9.32 \pm 0.14 \%$; $0.08 \pm 0.06 \%(N=3)$.

2.1.2.2. Method 2 - an abbreviated method, providing BHS2 SpECs Raw L. clavatum spores $(100 \mathrm{~g})$, were suspended in aqueous sodium hydroxide solution $\left(6 \% \mathrm{w} / \mathrm{w}, 520 \mathrm{~cm}^{3}\right)$ and heated at $80^{\circ} \mathrm{C}$ for $24 \mathrm{~h}$. The cooled mixture was filtered under vacuum, and the isolated SpECs stirred with $2 \mathrm{M} \mathrm{HCl}$ for $15 \mathrm{~min}$. After filtration, the particles were washed with deionized water $\left(3 \times 600 \mathrm{~cm}^{3}\right)$ until the washings became neutral. The extracted exines were then washed carefully with ethanol $\left(200 \mathrm{~cm}^{3}\right)$, then heated at reflux in ethanol $\left(530 \mathrm{~cm}^{3}\right)$ for $4 \mathrm{~h}$. The SpECs were filtered under vacuum, and finally washed with more ethanol $\left(2 \times 200 \mathrm{~cm}^{3}\right)$. The solid was left to dry under vacuum over phosphorus pentoxide to constant weight. Combustion elemental analysis: found C $57.48 \%$; $8.35 \%$;, $0.4 \%(N=1)$.

\subsubsection{Acid hydrolysed spores (AHS)}

Raw L. clavatum spores $(100 \mathrm{~g})$, were suspended in aqueous hydrochloric acid $(9 \mathrm{M})\left(450 \mathrm{~cm}^{3}\right)$ and heated at $90{ }^{\circ} \mathrm{C}$ for $1 \mathrm{~h}$. The cooled mixture was filtered, and the isolated SpECs washed with deionised water $\left(3 \times 600 \mathrm{~cm}^{3}\right)$ until the filtrate was neutral. The SpECs were filtered under vacuum and washed with ethanol $\left(3 \times 200 \mathrm{~cm}^{3}\right)$. The solid was left to dry under vacuum over phosphorus pentoxide to constant weight. Combustion elemental analysis found: Batch 1: C, $61.92 \%$; $7.58 \%$; N, $0.22 \%(N=1)$. Batch 2: C, $67.97 \%$; H $9.19 \%$;, $0.00 \%(N=1)$.

\subsubsection{Methylation of PHS SpECs (MePHS SpECs)}

To a stirred suspension of PHS SpECs $(10.0 \mathrm{~g})$ in acetone $\left(300 \mathrm{~cm}^{3}\right)$ was added anhydrous powdered potassium carbonate $(69.0 \mathrm{~g})$. Dimethyl sulfate $\left(24 \mathrm{~cm}^{3}\right)$ was added in portions over $10 \mathrm{~min}$ at room temperature. After the addition was completed, the mixture was heated at reflux for $3 \mathrm{~h}$, cooled to room temperature and filtered. The solid obtained was added to water $\left(500 \mathrm{~cm}^{3}\right)$ and stirred vigorously for 5-10 min to dissolve the potassium carbonate. A second filtration was followed by successive washing of the solid with deionised water $\left(3 \times 150 \mathrm{~cm}^{3}\right)$, ethyl acetate $\left(3 \times 150 \mathrm{~cm}^{3}\right)$, methanol $\left(3 \times 100 \mathrm{~cm}^{3}\right)$, and dichloromethane $\left(3 \times 100 \mathrm{~cm}^{3}\right)$, and it was then dried over $\mathrm{P}_{2} \mathrm{O}_{5}$ under vacuum to a constant weight. Combustion elemental analysis found: C, $72.84 \%$; H 8.27 $\%$;, $0.00 \%(N=1)$.

\subsection{Encapsulation of cod liver oil}

Briefly, the appropriate ratio (w/w) of $\omega-3$ oil and SpECs were mixed with gentle stirring using a glass rod or spatula for about $1 \mathrm{~min}$. The mixture was then placed optionally under a moderate vacuum (10-15 $\mathrm{mmHg}$ ) for around $10 \mathrm{~min}$ (Atkin et al., 2005; Barrier et al., 2011).

\subsection{UV irradiation}

A sample of either encapsulated or unencapsulated $\omega-3$ oil was spread thinly (approximately $3-4 \mathrm{~mm}$ deep) on a watch glass or Petri dish. The sample was placed underneath a UV lamp (Philips Original Home Solaria type HB 171/A, 220-230 volt $50 \mathrm{~Hz}$, $75 \mathrm{~W}$ possessing four Philips CLEO $15 \mathrm{~W}$ UV type 30 bulbs), at a distance of $13-15 \mathrm{~cm}$, and irradiated for the requisite length of time.
2.4. Peroxide value (PV) measurement (Paquot and Hautfenne, 1971; American Oil Chemists' Society, 2003)

A sample of $\omega-3$ oil $(1.00 \mathrm{~g})$ was mixed with chloroform $\left(10.0 \mathrm{~cm}^{3}\right)$, glacial acetic acid $\left(15.0 \mathrm{~cm}^{3}\right)$, and saturated aqueous potassium iodide $\left(1.0 \mathrm{~cm}^{3}\right)$. The mixture was shaken in a stoppered flask for $1 \mathrm{~min}$, and then left to stand in the dark for $5 \mathrm{~min}$ at room temperature (between $20-25^{\circ} \mathrm{C}$ ). The reaction was quenched by dilution with deionized water $\left(75.0 \mathrm{~cm}^{3}\right)$ and the liberated iodine was titrated against $0.01 \mathrm{M}$ standardised sodium thiosulfate solution using a starch indicator. PV, expressed in milliequivalents of active oxygen per mass unit of oil (mmeq. $\mathrm{kg}^{-1}$ ), is given by the formula:

$P V=\frac{V_{f}-V_{i}}{m} \times 10$

$V_{i}$ and $V_{f}$ are the initial and final volumes of the titrating solution $\left(\mathrm{cm}^{3}\right)$ and $m$ the mass of $\omega-3$ oil in the test sample $(\mathrm{g})$.

When the $\omega-3$ oil to be tested was encapsulated, either partially or fully within SpECs, the sample was first stirred in chloroform $\left(10.0 \mathrm{~cm}^{3}\right)$ for one minute to extract the $\omega-3$ oil. The $\omega-3$ oil and chloroform mixture was then separated from the SpECs by vacuum filtration, and the SpECs washed with glacial acetic acid $\left(15.0 \mathrm{~cm}^{3}\right)$ directly into the chloroform solution. The $\omega-3$ oil solution was then analysed as described above in this Section.

\subsection{Antioxidant protection over an extended timeframe}

The desired amount of SpECs was placed in a clear glass, stoppered bottle $\left(50 \mathrm{~cm}^{3}\right) . \omega-3$ Oil $\left(50 \mathrm{~cm}^{3}\right)$ was added, and the mixture was then shaken to disperse the SpECs. The bottles were left to stand in natural daylight at room temperature over the requisite timeframe, before PV measurements were taken as described above. During standing, the SpECs deposited at the bottom of the bottle. However, no further shaking or mixing was undertaken, and disturbance of the $\omega$-3 oil samples was minimised during sampling for the PV measurement. A control of $\omega-3$ oil $\left(50 \mathrm{~cm}^{3}\right)$ containing no SpECs was analysed alongside the samples containing SpECs. The PV of all samples was determined over the course of 10-12 weeks.

\subsection{Free-radical quenching using the 2,2-diphenyl-1-picrylhydrazyl $(D P P H)$ assay}

A stock solution of 2,2-diphenyl-1-picrylhydrazyl (DPPH) in ethanol $(1 \mathrm{mM})$ was prepared freshly each day to avoid any deterioration. To further minimise deterioration, all glassware, including the stock solution, sample tubes, and volumetric flasks were covered in aluminium foil and stored in the fridge when not in use or being analysed.

\subsubsection{Sample preparation}

SpECs $(10-15 \mathrm{mg})$ were weighed accurately into a sample tube covered in aluminium foil and DPPH stock solution $\left(1.0 \mathrm{~cm}^{3}, 1 \mathrm{mM}\right)$ was added. The sample was shaken for the allotted time to maximise the contact of the radical with SpECs. The SpECs were removed by filtration using a De Witt chamber, washed with a small amount of ethanol to ensure all the DPPH was removed, and the combined filtrate collected in a $50 \mathrm{~cm}^{3}$ volumetric flask. The flask was also covered in foil and made up to the mark with ethanol. The flask was stoppered and placed in a $+5{ }^{\circ} \mathrm{C}$ fridge until the analysis was conducted. A blank was prepared by also taking a stock solution $\left(1.0 \mathrm{~cm}^{3}\right)$ and diluting in a $50 \mathrm{~cm}^{3}$ volumetric flask. 


\subsubsection{UV/Vis measurements}

UV-vis absorption spectra were recorded on a Perkin-Elmer Lambda 10 ultraviolet-visible double-beam spectrophotometer with single-use polymethyl methacrylate cuvettes. For each time point the absorbance was measured in triplicate with the average being taken. The experiment was performed three times with the average radical quenched per gram of SpECs being calculated and plotted against time. The error bars were calculated based on the standard deviation of the radical quenched per gram of SpECs over the three runs.

\subsection{Statistical analyses}

Statistical analyses were performed using the 'XLMiner Analysis ToolPak' for Microsoft Excel. Statements regarding statistical significance between groups of data were determined either using two-way analysis of variance (ANOVA) or paired $t$-test assuming unequal variance as appropriate $(p<0.05)$.

\section{Results and discussion}

The protection of an $\omega-3$ oil to oxidation by encapsulation within SpECs at different $\mathrm{w} / \mathrm{v}$ ratios was investigated by either irradiation of the $\omega-3$ oil 'loaded' SpECs with UV light, or over an extended timeframe under ambient light conditions. Comparisons were made between SpECs extracted by different procedures (PHS, AHS, BHS; see Table 1), as well as irradiation of unencapsulated $\omega-3$ oil under the same conditions. The different extraction methods were explored to investigate if the extraction conditions had any influence on antioxidant activity of the SpECs and indication of changes to the functional group characteristics important to these properties. The peroxide value (PV) (Paquot and Hautfenne, 1971; American Oil Chemists' Society, 2003; Gotoh and Wada, 2006) was chosen as the principle means of determining the degree of oxidation of the oil, as it allowed a simple and rapid comparison of the antioxidant properties of the different type of SpECs and their formulations. In this assay, the level of peroxides and hydroperoxides formed during the initial stages of oxidation (Scott, 1997) are conveniently measured using a simple titrimetric protocol to give evidence of the incipient rancidity of the oil.

\subsection{Encapsulation of $\omega-3$ oil within SpECs}

Encapsulation of freshly purchased $\omega$-3 oil in the different types of $L$. clavatum SpECs was achieved using either the passive or vacuum filling method (Section 2.2) at a range of loading levels from 1:1 to $1: 6 \mathrm{w} / \mathrm{w}$ (SpECs:oil). At lower loadings (1:1 and 1:2 w/w) the $\omega-3$ oil appeared entirely encapsulated; however, at the higher ratios there was adhesion between the loaded SpECs and the product appeared as a paste (Fig. 1). This showed that there was $\omega-3$ oil on the outer surface of the SpECs and indicated incomplete encapsulation. L. clavatum SpECs have an outer diameter of $\sim 27 \mu \mathrm{m}$, a wall thickness of $\sim 2 \mu$

Table 1

SpECs used in this work, and a summary of their extraction method from raw spores of $L$. clavatum L.

\begin{tabular}{|c|c|}
\hline $\begin{array}{l}\text { SpECs } \\
\text { type }\end{array}$ & Summary of extraction method / modification \\
\hline PHS & (i) raw L. clavatum spores, acetone, $\Delta, 4 \mathrm{~h}$; (ii) $6 \% \mathrm{w} / \mathrm{v}$ aq. $\mathrm{KOH}, 80^{\circ} \mathrm{C}$, \\
\hline BHS1 & $\begin{array}{l}12 \mathrm{~h} \text {; (iii) } 85 \% \mathrm{H}_{3} \mathrm{PO}_{4}, 60^{\circ} \mathrm{C}, 5 \text { days. } \\
\text { (i) raw L. clavatum spores, acetone, } \Delta, 4 \mathrm{~h} \text {; (ii) } 6 \% \mathrm{w} / \mathrm{v} \text { aq. } \mathrm{KOH}, 80^{\circ} \mathrm{C} \text {, } \\
12 \mathrm{~h} \text {. }\end{array}$ \\
\hline BHS2 & (i) raw L. clavatum spores, $6 \% \mathrm{w} / \mathrm{v}$ aq. $\mathrm{NaOH}, 80^{\circ} \mathrm{C}, 12 \mathrm{~h}$. \\
\hline AHS & raw L. clavatum spores, $9 \mathrm{M} \mathrm{HCl}, 90^{\circ} \mathrm{C}, 1 \mathrm{~h}$ \\
\hline MePHS & PHS SpECs, $\mathrm{Me}_{2} \mathrm{SO}_{4}, \mathrm{~K}_{2} \mathrm{CO}_{3}$, acetone, $\Delta, 3 \mathrm{~h}$. \\
\hline
\end{tabular}
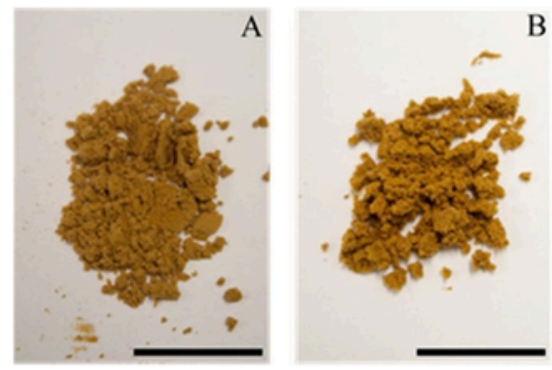

D
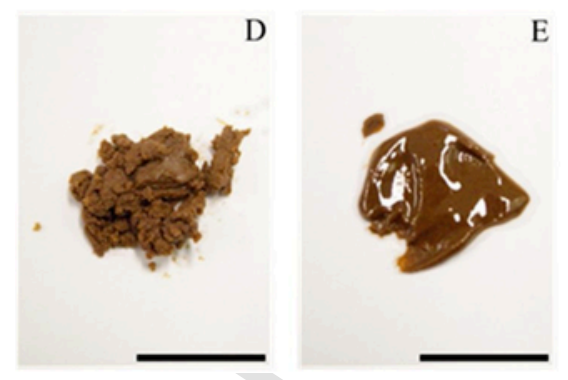

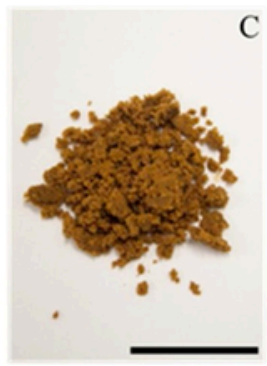

E

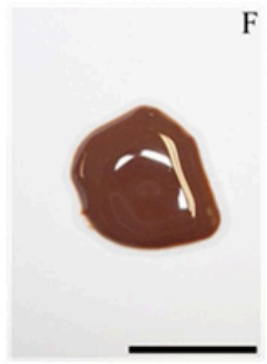

Fig. 1. Images of encapsulated cod liver oil at the following w/w ratios (PHS: oil); 1:1 (A), 1:2 (B), 1:3 (C), 1:4 (D), 1:5 (E), and 1:6 (F). Scale bar $=20 \mathrm{~mm}$.

$\mathrm{m}$, giving an internal volume for one capsule of $\sim 6370 \mu \mathrm{m}^{3}$. From mass, volume and close packing calculations the number of capsules in $1 \mathrm{~g}$ was estimated to be $354 \times 10^{6}$, giving a total internal volume of the capsules in $1 \mathrm{~g}$ of $2.25 \times 10^{12} \mu \mathrm{m}^{3}$. As the density of the $\omega-3$ oil is $0.92 \mathrm{~g} /$ $\mathrm{cm}^{3}\left(0.92 \times 10^{-12} \mathrm{~g} / \mu \mathrm{m}^{3}\right)$, this means the maximum volume of oil that could be fully encapsulated within $1 \mathrm{~g}$ of SpECs is $\sim 2.07 \mathrm{~g}$. These calculations are consistent the observations that at a $1: 2 \mathrm{w} / \mathrm{w}$ ratio or below the oil appeared fully encapsulated using the vacuum filling, whereas at higher levels the oil is also observed on the outside of the SpECs.

\subsection{UV irradiation of $\omega$-3 oil encapsulated in PHS SpECs}

The next part this study was to evaluate the protective properties of SpECs, either when the $\omega-3$ oil was either encapsulated within the cavity of the SpECs, or on its surface. It was decided that irradiation of samples with UV light over the range of loading levels would offer a convenient, accelerated way of determining the protection of the $\omega-3$ oil by the SpECs, as well as indicating whether any protection was due to simple light shielding or by acting as an antioxidant. Thus, samples of PHS SpEC-encapsulated $\omega-3$ oil were prepared at loading levels between 1:1 and 1:6 (SpECs:oil; w/w) using both passive and vacuum filling methods. After irradiation with UV light for the desired length of time, the $\omega-3$ oil was extracted and the PV determined (see Section 2.4). It is of note that an $\omega-3$ oil of good quality would have a PV of $<5$ mmeq. $\mathrm{kg}^{-1}$ (Freeman et al., 2001; Vinter, 1995), however it may develop characteristics of a rancid oil when exposed to air in a matter of hours. The control sample, i.e. $\omega-3$ oil that had not been encapsulated, had an average PV of 43.2 mmeq. $\mathrm{kg}^{-1}$ after $2 \mathrm{~h}$ of irradiation. At ratios of $1: 1$ and $1: 2$, where the $\omega-3$ oil was fully encapsulated by passive filling, the average PVs after exposure to UV irradiation were 18.4 and 22.9 mmeq. $\mathrm{kg}^{-1}$ respectively, showing that the $\omega-3$ oil had been protected against the UV irradiation. Next, the ratio of $\omega-3$ oil to SpECs was increased to levels where pastes and liquids were obtained (1:3 to $1: 6 \mathrm{w} / \mathrm{w}$, passive filling), i.e. there was clearly $\omega-3$ oil on the surface of the SpECs and not just inside. In these cases, the PVs obtained for all ratios were better than the control, and with the lowest proportion of SpECs (1:6 PHS SpECs:oil, w/w) the PV was as low as $10.4 \mathrm{mmeq} \cdot \mathrm{kg}^{-1}$. The PV values obtained with the $1: 1$ and $1: 2 \mathrm{w} / \mathrm{w}$ ratios seem to indicate that SpECs are less effective at protecting the oil than when pre- 
sent in the 1:3 and 1:4 w/w ratios (Fig. 2). At first glance this may seem counterintuitive, but could be explained by considering the fluidity of system when the excess oil is found on the outside of the SpECs (see section 3.1) rather than only being on the inside. We presume that in the former case, fatty acid hydroperoxides are able to diffuse in the bulk oil in and around the SpECs more freely, thus giving greater chances of their quenching by direct contact with the surface of the SpECs. With the $1: 1$ and $1: 2 \mathrm{w} / \mathrm{w}$ ratios the antioxidant activity of the SpECs is more restricted, giving only a "localized action" for the encapsulated oil.

The corresponding data obtained for vacuum filled SpECs showed no noticeable differences, indicating that the method of filling was not critical. It was clear from the results obtained that for either filling method at the concentrations of SpECs studied, the $\omega-3$ oil had been effectively protected. Therefore it was deduced that the SpECs could not be acting solely as a UV-shield in the ratios of 1:3 and above, where the $\omega-3$ oil is not fully encapsulated, and was concluded that SpECs were acting as an effective antioxidant.

It was decided next to conduct a study to see how the SpECs offered protection to the $\omega-3$ oil over time, to give a preliminary indication of the potential 'shelf-life' of an encapsulated oil. Initially, using a 1:1 w/ w PHS SpECs:oil ratio, the PV values were determined at various points over a period of $2.5 \mathrm{~h}$. The results showed that for the unprotected $\omega$ -
3 oil, the PV rose sharply after $1 \mathrm{~h}$ of irradiation (Fig. 3), indicating that the radical-induced chain reaction had been initiated. In contrast, after irradiation of PHS SpECs for $2 \mathrm{~h}$ the PV value was about the same as the unprotected $\omega-3$ oil after $1 \mathrm{~h}$. Even after $2.5 \mathrm{~h}$ the PV had only risen gradually, indicating clearly the protection provided by the PHS SpECs.

\subsection{Antioxidant protection over an extended timeframe}

To further investigate the indications that SpECs were acting as an antioxidant, as opposed to simply a UV shield, studies were carried out over an extended period using much lower quantities of SpECs compared with the $\omega-3$ oil. As described in Section 3.2, irradiation with a UV lamp leads to the onset of rancidity over the course of hours. These extended studies were carried out in natural daylight over weeks instead of hours, in order to give a better indicator of potential in a real life scenario. In these experiments the concentrations of SpECs used were $2 \%, 1 \%$, and $0.2 \% \mathrm{w} / \mathrm{v}$ relative to the $\omega-3$ oil, and both PHS and BHS1 SpECs were investigated.

The results showed that good protection was provided for both types of SpECs at all three concentrations (Fig. 4). Not surprisingly, the two samples that showed the better protection after 11 weeks, i.e. lowest measured PV, were when the SpECs were used at the $2 \%$ and $1 \% \mathrm{w} / \mathrm{v}$ concentrations. At both concentrations and for both types

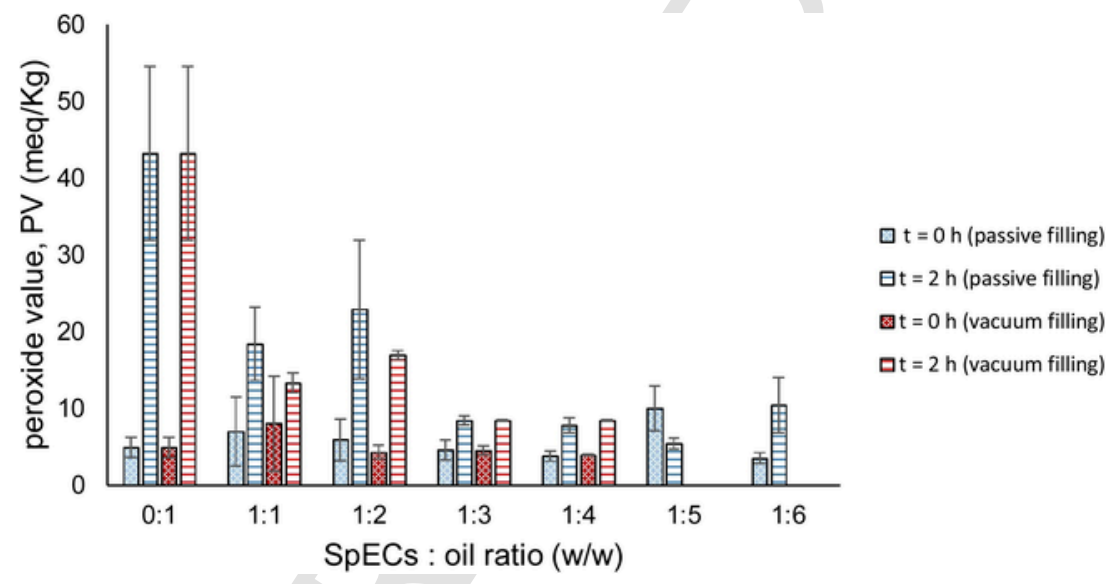

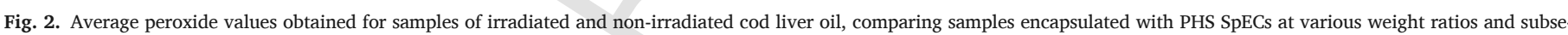

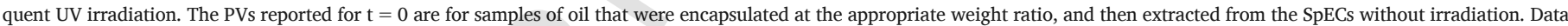
relating to both passive $(n=4)$ and vacuum $(n=2)$ encapsulation of oil is presented, and the error bars show the standard deviation.

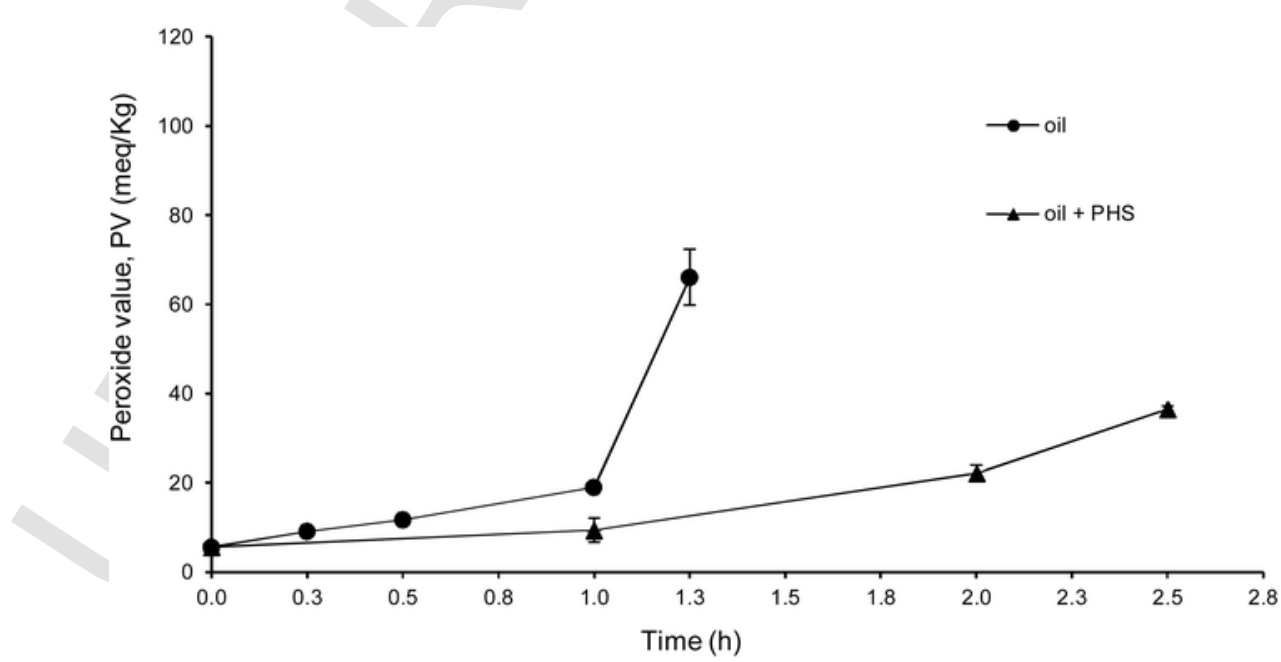

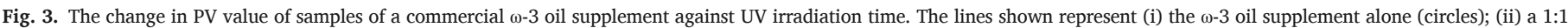

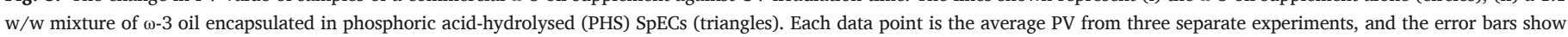
the standard deviation. 


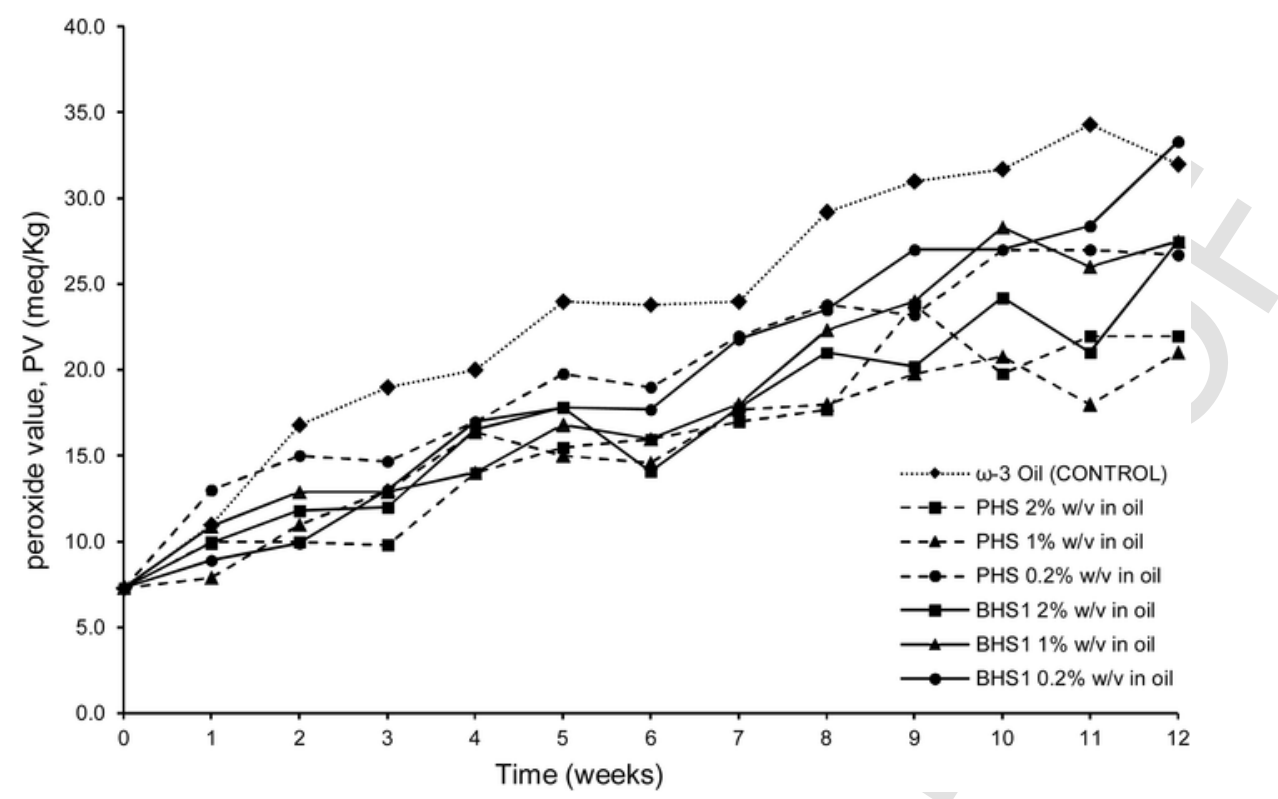

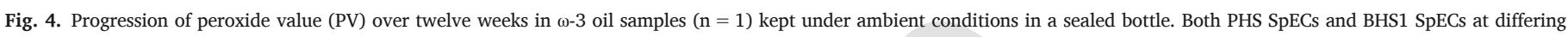

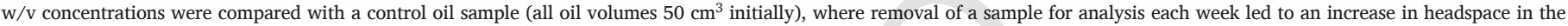
sample bottle.

of SpECs the lower PVs obtained compared to the 'oil only' data were statistically significant (one-tail $t$-test, $p<0.05$ ), but there was no significant differences comparing the performances of the PHS and BHS1 SpECs at the same concentrations (two-tail $t$-test, $p<0.05$ ). The samples that gave the worst protection, i.e. closest to the control, were the two samples that were tested at $0.2 \%$; however, it is important to note that even with this lower concentration, these results still demonstrated that less oxidation was taking place compared with the control. There was some indication that PHS SpECs may offer greater protection to the oil compared to the BHS1 SpECs at the same weight ratio to the oil. This would not be surprising as the BHS1 SpECs still contain the spore grain's intine layer (Diego-Taboada et al., 2012). Thus, BHS1 SpECs include cellulose ( $c a .10 \%$ by mass) as well as the sporopollenin, whereas the PHS SpECs are assumed to contain sporopollenin only. A more detailed study $(\mathrm{n}=4)$ was also performed using $1 \% \mathrm{w} / \mathrm{v}$ PHS SpECs only (data not shown), in which it was found that the same trend was followed as found in the single sample study shown in Fig. 4. Analysis of variance with this data showed the differences between the PV values of the oil with or without PHS SpECs $(1 \% \mathrm{w} / \mathrm{w})$ was statistically significant (two-way analysis of variance, $p<0.05$ ). Interestingly, it was also noted that the standard deviations for the average PVs for the $1 \% \mathrm{w} /$ $\mathrm{v}$ PHS SpECs in oil were much smaller compared with the corresponding controls. The relatively large variations in the PVs for the control samples revealed that the formation of peroxides in $\omega-3$ oils alone was rather sporadic during the initial stages of oxidation. These studies not only demonstrate that SpECs used at high concentrations offer a good level of protection to a sample of $\omega-3$ oil exposed to UV light, but also do so at much lower concentrations. Therefore, the SpECs can prolong potentially, the shelf-life of the $\omega-3$ oil compared with samples without added SpECs.

In the aforementioned experiments (Fig. 4), one sample had been analysed consecutively by removal of an aliquot of oil on a weekly basis. As the study progressed the head space in the sample bottle increased, and thus the amount of air present became larger as the experiment progressed. Therefore, in a subsequent study the experiments were repeated with $1 \% \mathrm{w} / \mathrm{v}$ PHS SpECs, with separate samples being analysed each week, such that the headspace in each sample bottle was constant throughout the course of the study. Comparing the initial PV at time zero and the PV at week ten, it can be seen that there was an overall increase by the end of the study in both the control and the PHS SpECs-containing samples (Fig. 5). Due to the limited quantity of oxygen present in the fixed headspace of the sample bottle, a small degree of initial oxidation (rise in PV) was to be expected. In subsequent weeks the PV value remained largely unchanged, albeit with a gradual increase, unlike the PV values presented in Fig. 4 which increased considerably over ten weeks. It was also noted that in this experiment and others, that the standard deviations in the control samples was much larger than that for the samples containing the PHS SpECs. The PVs of the control were fairly erratic over the course of the ten weeks, especially between week five and week ten. Nevertheless, comparison of the two sets of data (two-way analysis of variance, $p<0.05$ ) showed the differences were statistically significant, and this indicates that the SpECs provide a consistent level of antioxidant protection.

\subsection{UV irradiation of $\omega-3$ oil encapsulated in BHS1 SpECs, BHS2 SpECs and AHS SpECs}

If SpECs were to find use in a commercial application, then cost of production would be of great importance. As the spores come from a plant, production of the raw material is controlled largely by the scale of the harvest. However, the extraction process to obtain the SpECs would also have an important bearing on the final cost of the material. There are several procedures available for extraction of SpECs from raw plant pollens and spores. The general method used to obtain PHS SpECs dates from the times of Zetzsche (Zetzsche and Huggler, 1928; Zetzsche and Kalin, 1931) and Shaw (Shaw, 1971). Methods using phosphoric acid are typically more expensive as the cost of the acid is not insignificant and the length of heating is often long. Some groups have investigated shorter extractions, but still using phosphoric acid (Gonzalez-Cruz et al., 2018; Mundargi et al., 2016). To avoid the use of phosphoric acid entirely, simpler and cheaper methods of obtaining SpECs capsules simply omit that step entirely. Thus, whilst still providing clean microcapsules, base-hydrolysis alone gave a product (BHS1 SpECs) in which most of the inner polysaccharide or 'intine' layer remains (Diego-Taboada et al., 2012). An even more abbreviated base hydrolysis, omitting the acetone pre-treatment and using the cheaper $\mathrm{NaOH}$, yielded BHS2 SpECs, and a one-step extraction using hot 9 M HCl gave acid-hydrolysed spore SpECs (AHS SpECs) after just one 


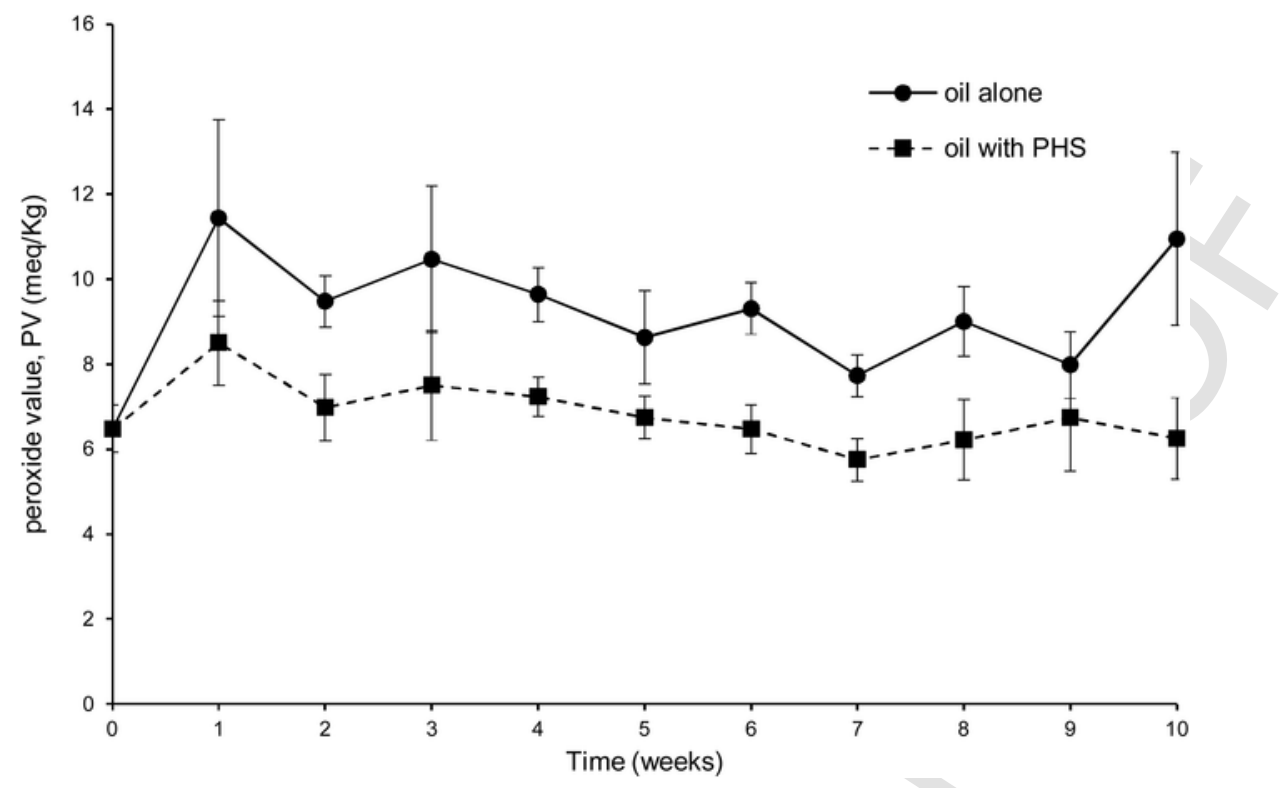

Fig. 5. Progression of average peroxide values $(n=4)$ over ten weeks for samples of $\omega-3$ fish oil containing $1 \%$ w/v PHS SpECs in sample bottles with constant headspace volume.

hour of heating. Either of these alternative methods (Table 1) could offer a cost and time advantage compared with extraction of the SpECs via the original three-step procedure using phosphoric acid. The question posed now was whether the method of extraction affected the antioxidant properties of the SpECs. In addition, it was of interest to see if the base extracted SpECs lost antioxidant properties, as observed by Smirnova and co-workers (Smirnova et al., 2012) for the more fragile sporopollenin extracts from $N$. tabacum $\mathrm{L}$.

To compare the different types of SpECs, samples were prepared accordingly (see Section 2.1) and the encapsulation and irradiation experiments conducted as usual (Sections 3.1 and 3.2). Firstly, a smaller study was conducted in which the BHS1 SpECs to oil weight ratio was varied. The PV obtained at $\mathrm{w} / \mathrm{w}$ ratios of $1: 2,1: 3$, and $1: 4$ after $2 \mathrm{~h}$ exposure to UV irradiation were 12.4, 8.6, and $14.2 \mathrm{mmeq} . \mathrm{kg}^{-1}$, respectively (Fig. 6), and compared with the data for PHS SpECs obtained earlier (Fig. 2).

Comparing these data it appears that the presence of the polysaccharide 'intine' layer has little effect on the PV values, as might be expected since it is mostly cellulosic. Moreover, no clear reduction in antioxidant capacity was observed after base treatment, as may have been predicted by the work of Smirnova and co-workers (Smirnova et al., 2012). The SpECs extracted using the two shortest procedures were evaluated next; the one-step base extraction, providing BHS2 SpECs, and the hydrochloric acid extraction providing AHS SpECs. As before, a 1:1 w/w oil:SpECs ratio was chosen, but this time the analysis conducted over a period of several hours using UV irradiation. The results of this study revealed that the $\omega-3$ oil samples encapsulated in either type of SpECs showed a similar and much slower rise in PV between one and three hours when compared with the unencapsulated $\omega-3$ oil control sample (see Fig. 7). Comparing the overall results from these experiments, it revealed that the PV value of oil encapsulated in any type of SpECs increases very slowly during the timeframe studied, compared with the PV of the control $\omega-3$ oil samples which rose markedly during the same period. Thus, it appears that it does not matter which extraction process is used; the SpECs offer the same or similar level of antioxidant protection irrespective of the extraction process used on the raw spores. However it was noted, comparing data in Figs. 3 and 7, that the rise in PV of the $\omega-3$ oil controls did not show repeatable behaviour. The data for the $\omega-3$ oil control collected during the PHS study showed a sharp upturn in PV at $1.25 \mathrm{~h}$ irradiation (Fig. 3), whereas

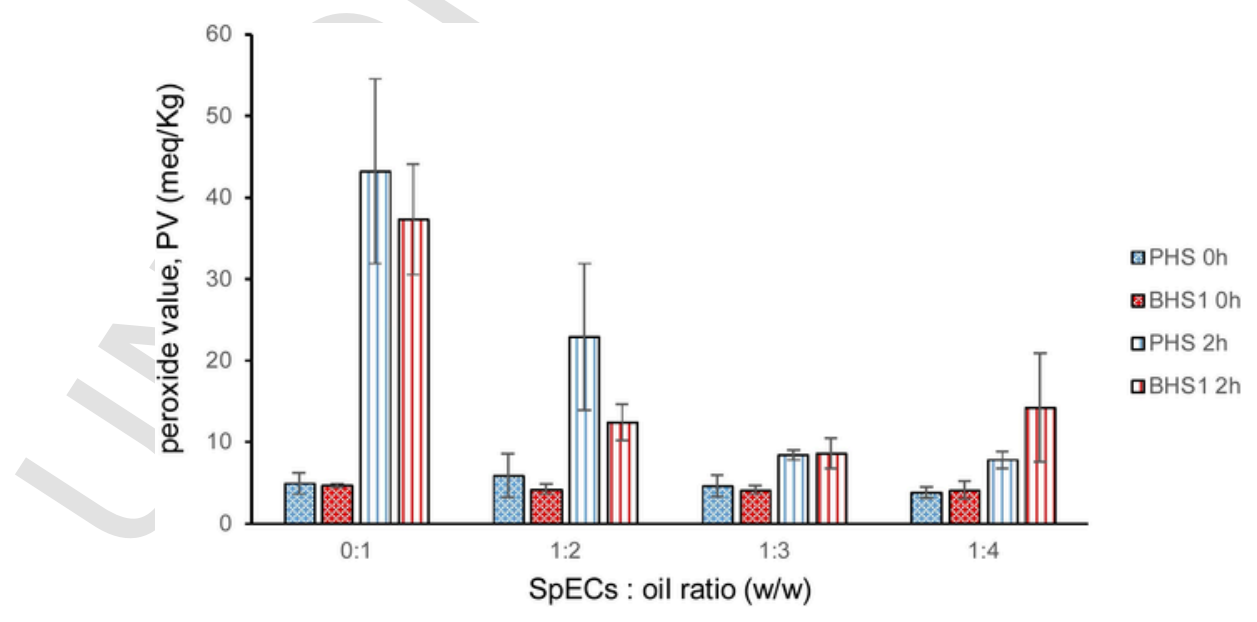

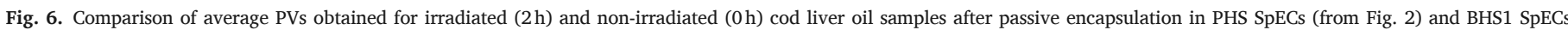

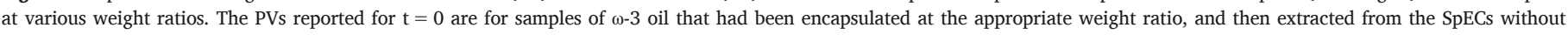
irradiation. Error bars shown are the standard derivation of the average. 


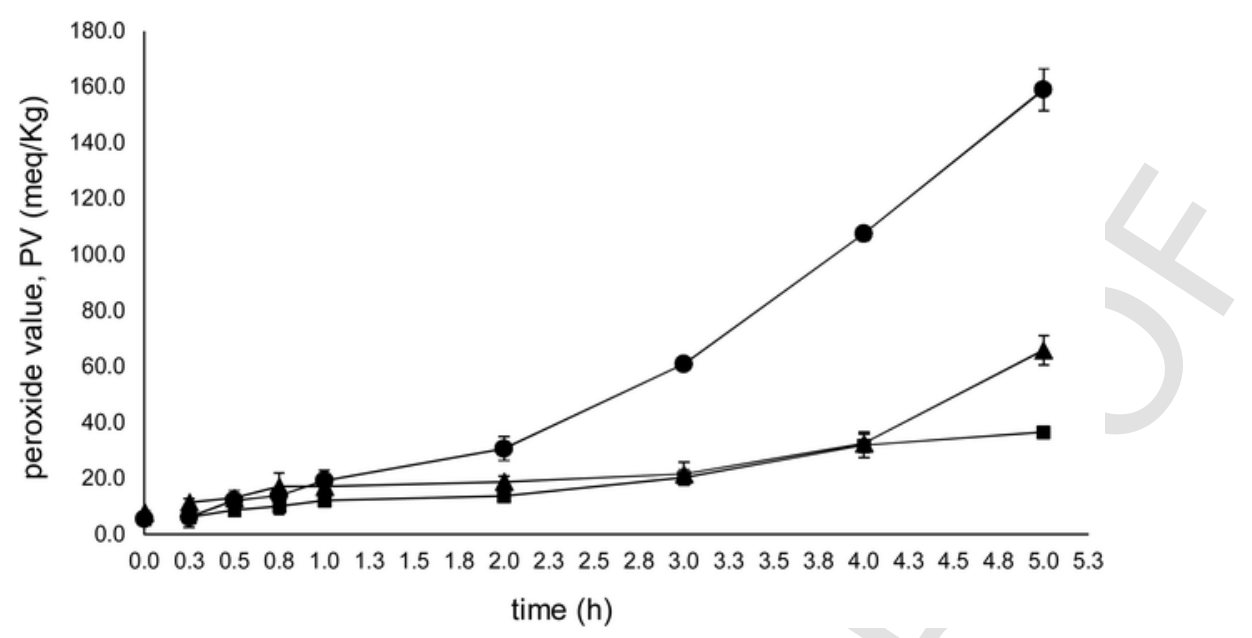

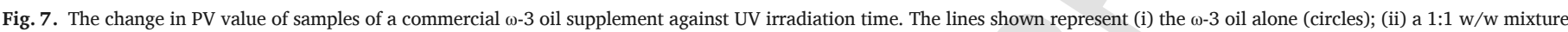

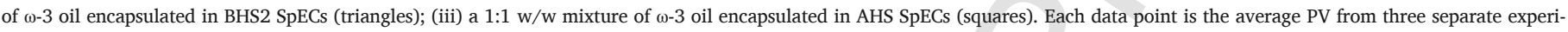
ments, and the error bars show the standard deviation.

the rise in PV for the control $\omega-3$ oil was more gradual in the BHS2 / AHS SpECs study (Fig. 7). The PV values of the $\omega-3$ oil samples were always tested daily before experimentation to ensure that they had not started to rancify ( $c f$. Materials and Methods). The explanation for these differences are likely due to the fact that the batches of oil for each experiment (Fig. $3 v s$ Fig. 7) were different, and the experiments were conducted under ambient conditions on different days by different researchers. There was also no indication as to how long the bottles had been on the shelf of the stores from which they were bought. However, this was not considered to be problematic, as in all cases the rise of the $\mathrm{PV}$ value in the oil control samples were greater and faster than when the same oil was encapsulated in a concurrent experiment. It was observed also that once the PVs had risen above a certain level, even with due care and attention, the standard deviation in repeat experiments was much greater for the unencapsulated oil samples. Thus, whilst the absolute PVs were subject to some batch and operator variation, the trends were always clear and consistent: SpECs offered antioxidant protection of the $\omega 3$-unsaturated oil.

\subsection{Batch variability of PHS and BHS SpECs}

Since the activity of an antioxidant is related to its chemical structure, the same antioxidant activity is to be expected 'from molecule to molecule'. The previous experiments indicated some variation in antioxidant activity of SpECs extracted in different ways, but as the material is a natural and amorphous polymer it was considered equally important to test if there was any variability of the antioxidant properties between different batches of SpECs when the extraction methods had been performed in an identical fashion by the same operator. Thus, experiments were conducted using separate batches of PHS SpECs and BHS1 SpECs. Extended timeframe experiments were carried out as described in Section 3.3, using 2\%, $1 \%$ and $0.2 \% \mathrm{w} / \mathrm{v}$ ratio of SpECs to oil. All experiments were run concurrently, using the same oil samples as control. Within both batches, the $1 \%$ and $2 \% \mathrm{w} / \mathrm{w}$ PHS SpECs:oil ratios showed similar levels of inhibition of $\omega-3$ oil oxidation, with $0.2 \%$ $\mathrm{w} / \mathrm{v}$ offering least protection (Fig. 8A). For both batches of PHS SpECs at all loadings, the differences between the PV values obtained from the oils with or without SpECs were statistically significant. However, it was also found that there was also a significant difference between the two batches of PHS SpECs at the 1 and $2 \% \mathrm{w} / \mathrm{v}$ loading (two-tail $t$-test, $p<0.05$ ). As sporopollenin is a natural product, the conditions in which the plants are grown, the nutrients and the amount of sunlight the plant receives could all play a role in the composition and quan- tity of the sporopollenin present in the spores. However, both of these batches were obtained from the same source of L. clavatum spores and so this factor is unlikely to explain the differences observed. The batch comparison experiments were repeated in an identical fashion using BHS1 SpECs. As can be seen in Fig. 8B, data from different batches of BHS1 SpECs at 1 and $2 \% \mathrm{w} / \mathrm{v}$ looked very similar, though the data suggested there may be a possible variation between batches at the $0.2 \%$ $\mathrm{w} / \mathrm{v}$ loading. However, in all cases the differences between PV values obtained from the oils with and without BHS1 SpECs at all loadings were statistically significant, and further analysis also showed that there were no statistically significant differences between the data from the two batches at the same $\mathrm{w} / \mathrm{v}$ loading level (two-tail $t$-test, $p<0.05$ ). As these samples had not undergone treatment with phosphoric acid it may be that the variation between the batches of PHS SpECs is due to differences arising from that specific step of the extraction. Whilst the primary aim of the phosphoric acid extraction step is to remove the polysaccharide intine layer, it also plays part in the quantity of functional groups on the surface of the extracted SpECs and can lead to variable colour changes in the SpECs if the temperature is not controlled carefully. Possible surface functional group changes during this treatment are dehydration of alcohols, formation of lactones, and possibly formation of diaryl ethers formed by cross linking of phenols. The washing procedure to get a clear filtrate at the end of the phosphoric acid extraction step is also long and bothersome. It is therefore likely that it was this stage of the process which led to the differences observed in the antioxidant tests and means that the 'identical' extraction process were not so similar as expected.

\subsection{Free-radical quenching using 2,2-diphenyl-1-picrylhydrazyl (DPPH) assay}

Quenching of the 2,2-diphenyl-1-picrylhydrazyl (DPPH) radical has been used widely as an antioxidant assay (Sharma and Bhat, 2009), and it was thought that its use in this project would assist in understanding more about the mechanism by which SpECs act as an antioxidant. The DPPH method relies on the spectrophotometric determination of the DPPH concentration change resulting from the reaction with an antioxidant such as a phenol, commercial antioxidant, or plant extract etc. Using the measured absorbance and known concentration of a blank, the $\varepsilon$ value for DPPH-H can be calculated using the Beer-Lambert law. The concentration of DPPH radicals in the filtrate, after contact with an antioxidant, can be calculated using the absorbance of the filtrate and $\varepsilon$. The difference in DPPH radical concentrations in the fil- 

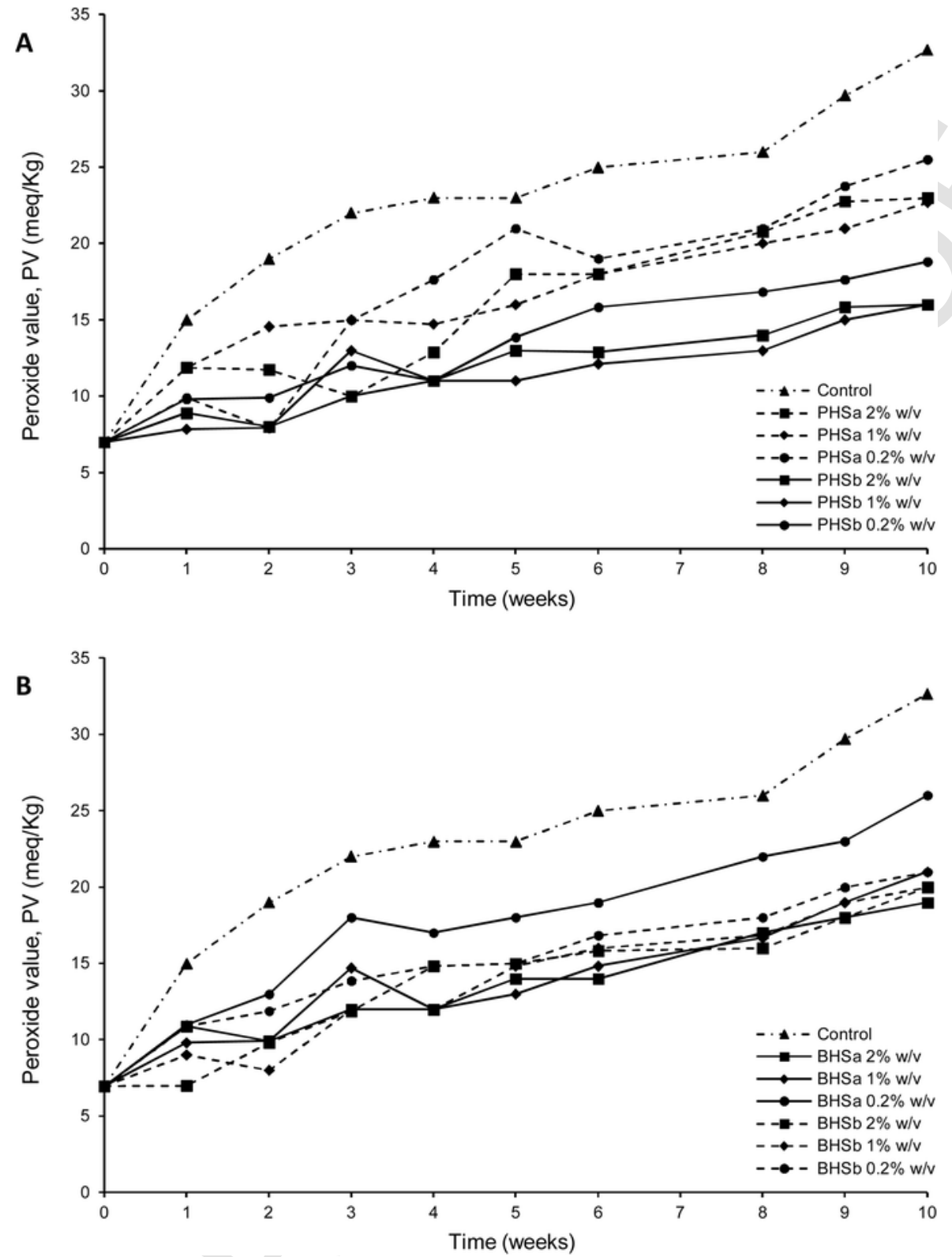

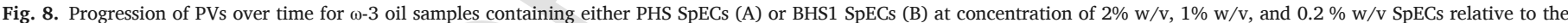
$\omega-3$ oil. On each graph two identically extracted batches of SpECs, labelled a or b, are compared.

trate from the blank is then regarded as the amount of radical quenched. As the mass of added antioxidant used is known, the theoretical quantity of radical quenched per gram of antioxidant can thus be determined. Early studies based on this assay used soluble substrates, but the method was adapted successfully by Serpen and co-workers (Serpen et al., 2007) for insoluble materials. Application of this DPPH radical quenching method to PHS SpECs was therefore undertaken. The results obtained showed that there was an initial rise in the amount of radical being quenched during the first $5 \mathrm{~min}$ of the assay, followed by a gradual increase up to one hour whereupon a steady state was reached (see Fig. 9). At steady state this revealed that the SpECs had quenched $1.48 \pm 0.13 \mu \mathrm{mol}$ of DPPH radicals per gram of SpECs. This gave direct evidence that the SpECs can protect $\omega-3$ oils by acting as an antioxidant. Since this initial study was aimed at scoping the potential of different SpECs preparations, it has not yet been extended to determine the amount secondary oxidation in SpECs. The $p$-anisidine test (Hamilton et al., 1998) could, for example, be used to ob-

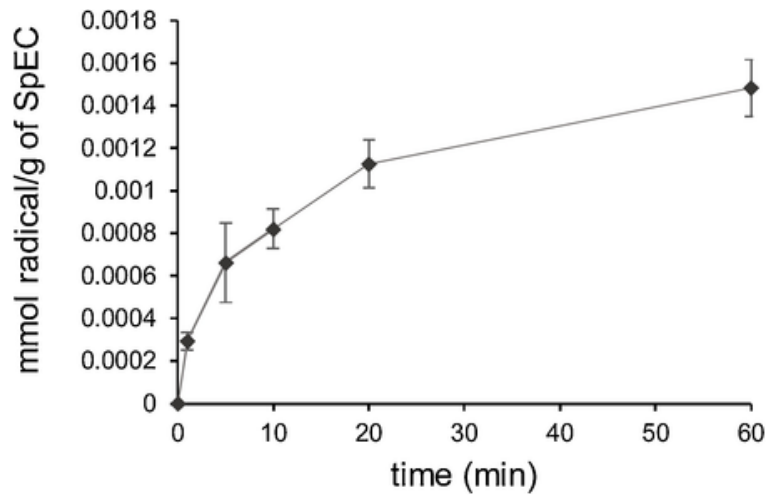

Fig. 9. Time profile of the mmol of DPPH radicals quenched per gram of PHS SpECs over the course of $60 \mathrm{~min}(\mathrm{n}=5)$. 
tain an anisidine value (AV), and from that a Totox Value (AV + 2PV) determined.

\subsection{Comparison of the antioxidant properties of PHS SpECs with BHT and MePHS SpECs}

Whilst the practical protective properties of SpECs had been demonstrated, and the DPPH assays showing that SpECs were effective at quenching radicals, it was of interest to obtain further evidence that it was the phenolic groups specifically that give rise to the SpECs' protective antioxidant properties. Previous work, using a modified Boehm titration method, had estimated that the amount of available phenolic groups on the PHS SpECs was between 0.30 and $0.35 \mathrm{mmol} / \mathrm{g}$ (Diego-Taboada et al., 2012). This data meant it was possible to use the same ratio of moles of small molecule phenol antioxidant per gram of oil for the fairest comparison with the results obtained using SpECs. Thus, mirroring the $1: 1 \mathrm{w} / \mathrm{w}$ ratio loading of PHS SpECs and oil, the irradiation experiments were repeated with 2,6-di-tert-butyl-4-methylphenol (BHT), in which $0.30 \mathrm{mmol}$ of added phenol was used per gram of $\omega-3$ oil. Inspection by eye suggested that the BHT was not soluble in the $\omega-3$ oil, but as the SpECs are not soluble either this was considered to be a reasonable model for comparison. Nevertheless, the solids were ground as finely as possible, and dispersed evenly in the $\omega-3$ oil at the start of each experiment. Irradiation of the $\omega-3$ oil containing $0.3 \mathrm{mmol} / \mathrm{g}$ BHT and subsequent titration of the extracted $\omega-3$ oil, produced a set of PV values across $2.5 \mathrm{~h}$ which were quite similar to those obtained for the PHS SpEC encapsulated $\omega-3$ oil (Fig. 10). This result gave further weight to the argument that the antioxidant properties of SpECs are mediated by phenolic groups contained in the sporopollenin polymer, and that their effectiveness per mole of phenol is similar to known small molecule antioxidant.

It is known that the surface of SpECs contains a range of functional groups, which can be chemically modified (Diego-Taboada et al., 2012). Therefore, the next logical step was to see whether "capping" the phenolic groups blocked SpECs from performing an antioxidant role. Consequently, modified PHS-SpECs were prepared in which the free hydroxyl functions (alcohol, phenol and carboxylic acid groups) were methylated (dimethyl sulfate and potassium carbonate) to form the corresponding methyl ethers and esters. The $\omega-3$ oil was encapsulated in the methylated PHS SpECs (MePHS SpECs) in a 1:1 w/w ratio, and the irradiation and titration analyses conducted as aforementioned. Contrary to the performance of the PHS SpECs and BHT, the onset of $\omega-3$ oil rancidity using MePHS SpECS was found to be comparable to that of the free $\omega-3$ oil; none of the protective properties of the capsules were retained upon methylation (Fig. 10). This finding further indicates that the presence of the phenols play a key role in the SpECs' antioxidant properties.

\subsection{Comparison of the antioxidant properties of BHS SpECs with ferulic,} coumaric, and 4-methoxy cinnamic acids

As discussed in the Introduction, sporopollenin, the polymer which makes up the plant spore's exine, is both physically and chemically robust. The polymer has a complex structure that has not yet been elucidated fully, even though a great deal is known from various spectroscopies and degradation studies (Barrier, 2008). The latter reveals that low molecular weight acids are released from oxidative degradation of sporopollenin, amongst which are 4-hydroxy-3-methoxycinnamic acid (ferulic acid) and 4-hydroxycinnamic acid (coumaric acid). As the performance of SpECs compared favourably to the known small molecule antioxidant BHT on a mole for mole basis, this naturally led us to investigate these substituted cinnamic acid derivatives in the same fashion. Thus, using the same methodology as for BHT, comparison was made between BHS SpECs, ferulic acid, coumaric acid, and 4-methoxycinnamic acid (Fig. 11). The latter, with a methylated oxygen function was included to make comparison with the methylated SpECs, MePHS SpECs, investigated earlier (Section 3.7).

The results obtained revealed that the performance of ferulic acid, on a mole for mole phenol basis, compared favourably to the performance of BHS SpECs even over an extended time of five hours irradiation (Fig. 11). In comparison, the monosubstituted coumaric acid matched the SpECs up two hours but was increasingly less efficient between two to five hours of irradiation. In contrast, the protection offered by 'capped' phenol, 4-methoxycinnamic acid, was so inefficient that after two hours the PV value was already around four times greater than for the other samples.

\section{Conclusions}

Sporopollenin exine capsules (SpECs) are monodisperse particles of approximately $27 \mu \mathrm{m}$ in diameter and with a wall thickness of around $2 \mu \mathrm{m}$. The exine material is made of sporopollenin, a biomaterial comprising primarily of polymerised and cross-linked lipids, and which also contain phenol, alcohol, and carboxylic acid groups on their surface (Mackenzie et al., 2015). These exine capsules may be extracted from spores of L. clavatum L. via several methods, which may (using

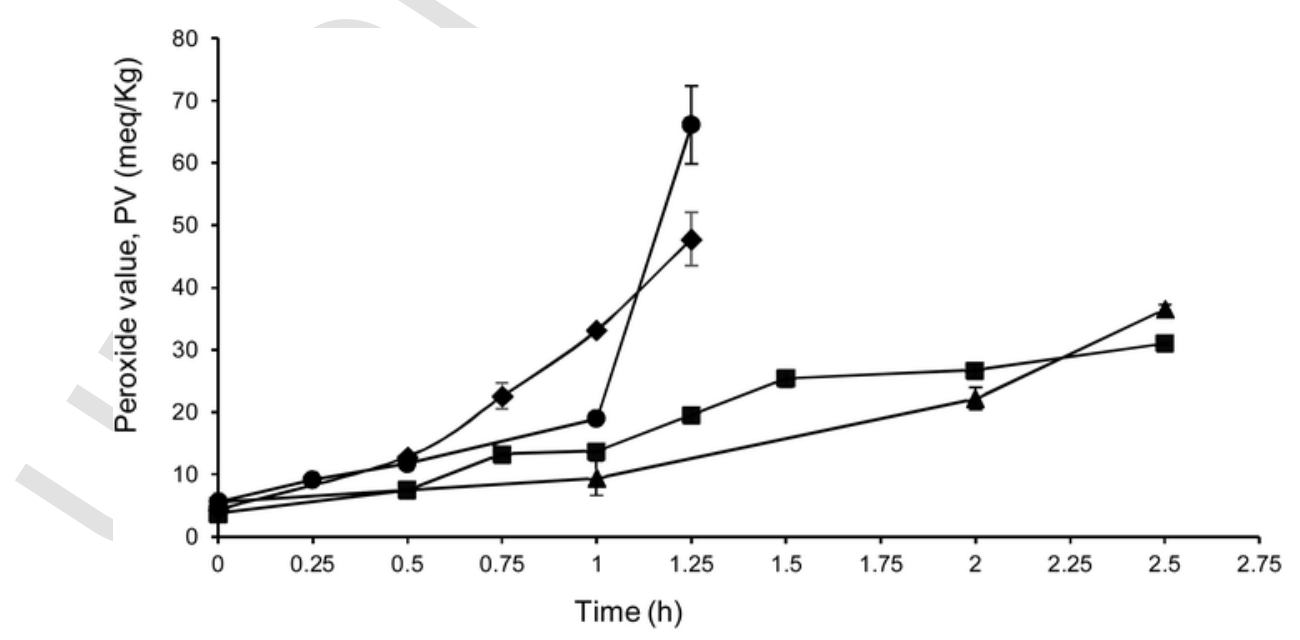

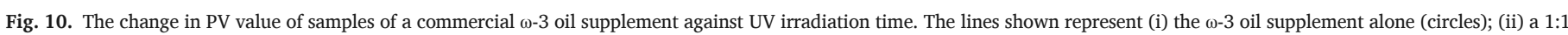

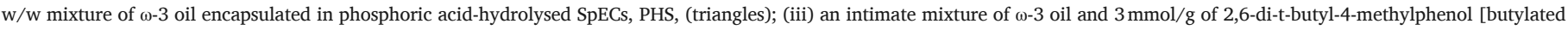

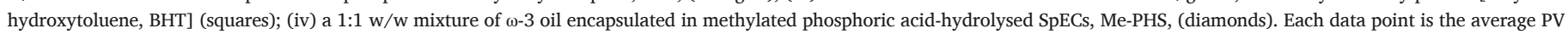
from three separate experiments, and the error bars show the standard deviation. 


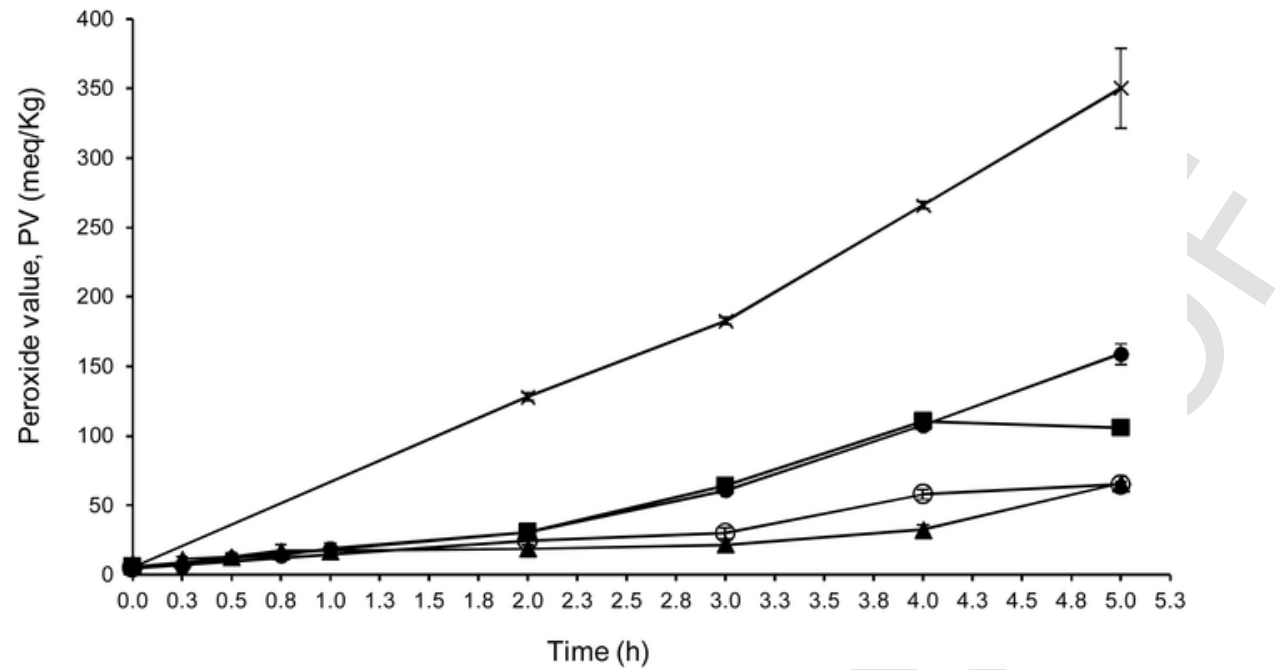

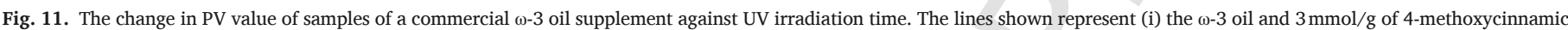

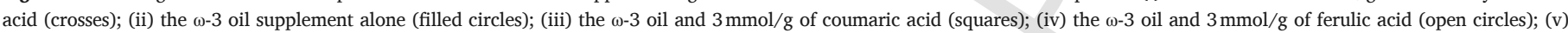

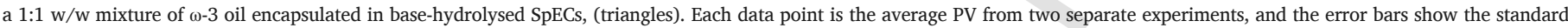
deviation.

hot acid) or may not (using hot alkali) remove the inner 'intine' layer of polysaccharide (Diego-Taboada et al., 2012).

Results revealed herein show that SpECs from L. clavatum L., produced by a range of extraction methods, are capable of protecting an $\omega-3$ oil from accelerated (UV irradiation) or normal (ambient light) oxidation processes at a range of $\mathrm{w} / \mathrm{w}$ ratios, even when the oil is in vast excess. In this work it has also been shown that the practical antioxidant activity of SpECs is not exerted by light screening, rather through the non-hydrolysable phenolic groups which are part of the polymeric structure of sporopollenin from L. clavatum $\mathrm{L}$. This has been investigated through a range of experiments, namely DPPH radical quenching, and comparisons with ferulic and coumaric acids, and BHT. Total removal of the antioxidant activity is also observed when the phenol groups on the SpECs are thoroughly methylated, as was observed if $p$-methoxycinnamic acid was used in place of coumaric ( $p$-hydroxycinnamic) acid. The commercially-sourced $\omega-3$ oil chosen for this study does contain small amounts of the lipid soluble $\alpha$-tocopherol; hence, whether SpECs provided an additive or synergistic antioxidant effect remains unanswered at this time pending conclusion of on-going studies

The naturally derived SpECs, which have been shown previously to be protein free and non-allergenic, may therefore prove to be a useful natural alternative to small synthetic molecules for the protection of $\omega-3$ unsaturated fatty acid-containing compounds towards oxidation. The ability of the SpECs to provide protection from peroxide-induced, free radical-mediated oxidation processes, may also be extended to materials other than unsaturated fatty acids. It is also pertinent to note at this juncture, complementary recent work has been reported showing potent photoprotective properties of SpECs (Bailey et al., 2019). This work revealed that the light-sensitive macrocyclic antibiotic marinomycin $\mathrm{A}$ can be protected from photoisomerisation (and hence inactivation) by encapsulation within SpECs. Thus, the combined (or individual) antioxidant and light screening properties of SpECs offer a range of new ways of protecting light sensitive compounds using a bio-inspired material. CRediT authorship contribution statement

Matthew J. Thomasson: Methodology, Investigation, Formal analysis, Visualisation. Alberto Diego-Taboada: Methodology, Investigation, Formal analysis. Sylvain Barrier: Methodology, Investigation, Formal analysis. Jules Martin-Guyout: Investigation, Formal analysis, Visualisation. Elizabeth Amedjou: Investigation, Formal analysis,
Visualisation. Stephen L. Atkin: Conceptualization, Funding acquisition. Yves Queneau: Methodology, Writing - review \& editing. Andrew N. Boa: Supervision, Methodology, Writing - original draft. Grahame Mackenzie: Conceptualization, Funding acquisition, Methodology, Supervision, Writing - review \& editing.

\section{Declaration of Competing Interest}

S.L.A. and G.M. are directors of Sporomex Ltd, which owns patents relevant to the work presented in this paper. For all other authors, there are no conflicts of interest.

\section{Acknowledgements}

We thank Sporomex Ltd. (for M.J.T., A.D.T.) and the University of Hull (for S.B., J.M.G., E.A.) for funding this work. We are also grateful for the advice and support from the contractors (funded organisations) involved in the 2008-2011 DEFRA Link programme "Micro-encapsulation of active ingredients using renewable material from rye and maize pollens - LK0837": Croda International PLC, The Boots Company PLC, Frontier Agriculture and Sporomex Ltd. Finally, we thank Dr A. D. Walmsley (University of Hull) for advice regarding the statistical analyses performed.

\section{References}

Allen, J.C., Hamilton, R.J. (Eds.), 1994. Rancidity in Foods, third edSpringer, US.

American Oil Chemists' Society, 2003. Peroxide value - acetic acid-chloroform method. AOCS official method Cd 8-53. Official Methods and Recommended Practices of the AOCS. AOCS Press, Champaign Illinois.

Atkin, S. L., Beckett, S. T., Mackenzie, G., 2005. Dosage form comprising an exine coating of sporopollenin or derivatized sporopollenin. Patent WO2005000280.

Atkin, S. L., Beckett, S. T., Mackenzie, G., 2007. Uses of sporopollenin: antioxidant properties. Patent WO2007012856.

Atkin, S.L., Barrier, S., Cui, Z., Fletcher, P.D.I., Mackenzie, G., Panel, V., Sol, V., Zhang, X., 2011. UV and visible light screening by individual sporopollenin exines derived from Lycopodium clavatum (club moss) and Ambrosia trifida (giant ragweed). J. Photochem. Photobiol. B Biol. 102, 209-217. https://doi.org/10.1016/j.jphotobiol.2010. 12.005 .

Bai, Z.Y., Zhang, S.M., Liu, X., Sheng, S., Wu, F.A., Wang, J., 2019. Generation of alpha-linolenic acid ethyl Ester microparticles from silkworm pupae oil by microfluidic droplet. Waste Biomass Valori. 10, 3781-3791. https://doi.org/10.1007/s12649-018 00572-y.

Bailey, C.S., Zarins Tutt, J., Agbo, M.O., Gao, H., Diego-Taboada, A., Gan, M., Hamed, R.B., Abraham, E., Mackenzie, G., Evans, P.A., Goss, R.J.M., 2019. A natural solu- 
tion to photoprotection and isolation of the potent polyene antibiotic, Marinomycin A. Chem. Sci. 10, 7549-7553. https://doi.org/10.1039/C9SC01375J.

Barrier, S., 2008. Physical and Chemical Properties of Sporopollenin Exine Particles, PhD Thesis. University of HullIn: https://hydra.hull.ac.uk/assets/hull:6412a/content.

Barrier, S., Rigby, A.S., Diego-Taboada, A., Thomasson, M.J., Mackenzie, G., Atkin, S.L., 2010. Sporopollenin exines: a novel natural taste masking material. LWT-Food Sci. Technol. 43, 73-76. https://doi.org/10.1016/j.lwt.2009.07.001.

Barrier, S., Diego-Taboada, A., Thomasson, M.J., Madden, L., Pointon, J.C., Wadhawan, J.D., Beckett, S.T., Atkin, S.L., Mackenzie, G., 2011. Viability of plant spore exine capsules for microencapsulation. J. Mater. Chem. 21, 975-981. https://doi.org/10.1039/ C0JM02246B.

Diego-Taboada, A., Cousson, P., Reynaud, E., Huang, Y., Lorch, M., Binks, B.P., Queneau, Y., Boa, A.N., Atkin, S.L., Beckett, S.T., Mackenzie, G., 2012. Sequestration of edible oil from emulsions using new single and double layered microcapsules from plant spores. J. Mater. Chem. 22, 9767-9773. https://doi.org/10.1039/C2JM00103A.

Diego-Taboada, A., Maillet, L., Banoub, J.H., Lorch, M., Rigby, A.S., Boa, A.N., Atkin, S.L., Mackenzie, G., 2013. Protein free microcapsules obtained from plant spores as a model for drug delivery: ibuprofen encapsulation, release and taste masking. J. Mater. Chem. B 1, 707-713. https://doi.org/10.1039/C2TB00228K.

Freeman, I. P., Van Lookeren, G. J., Padley, F. B., Polman, R. G., 2001. Marine/vegetable oil blend and products made therefrom. Patent EP0304115A2.

Gonzalez-Cruz, P., Uddin, M.J., Atwe, S.U., Abidi, N., Gill, H.S., 2018. Chemical treatment method for obtaining clean and intact pollen shells of different species. ACS Biomater. Sci. Eng. 4, 2319-2329. https://doi.org/10.1021/acsbiomaterials.8b00304.

Gotoh, N., Wada, S., 2006. The importance of peroxide value in assessing food quality and food safety. J. Am. Oil Chem. Soc. 83, 473-474. https://doi.org/10.1007/s11746-0061229-4.

Hamilton, R.J., Kalu, C., McNeill, G.P., Padley, F.B., Pierce, J.H., 1998. Effects of tocopherols, ascorbyl palmitate, and lecithin on autoxidation of fish oil. J. Am. Oil Chem. Soc. 75, 813-822. https://doi.org/10.1007/s11746-998-0231-4.

Herminghaus, S., Gubatz, S., Arendt, S., Wiermann, R., 1988. The occurrence of phenols as degradation products of natural sporopollenin - a comparison with synthetic sporopollenin. Z. Naturforsch. C43, 491-500. https://doi.org/10.1515/znc-1988-7-803.

Leja, M., Mareczek, A., Wyżgolik, G., Klepacz-Baniak, J., Czekońska, K., 2007. Antioxidative properties of bee pollen in selected plant species. Food Chem. 100, 237-240. https://doi.org/10.1016/j.foodchem.2005.09.047.

Li, F.-S., Phyo, P., Jacobowitz, J., Hong, M., Weng, J.K., 2019. The molecular structure of plant sporopollenin. Nat. Plants 5, 41-46. https://doi.org/10.1038/s41477-018-03307.

Mackenzie, G., Boa, A.N., Diego Taboada, A., Atkin, S.L., Sathyapalan, T., 2015. Sporopollenin, the least known yet toughest natural biopolymer. Front. Mater. 2, 66. https:// doi.org/10.3389/fmats.2015.00066.

Meuter-Gerhards, A., Schwerdtfeger, C., Steuernagel, S., Wilmesmeier, S., Wiermann, R., 1995. Studies on sporopollenin structure during pollen development. Z. Naturforsch. C50, 487-492. https://doi.org/10.1515/znc-1995-7-804.

Mikhael, A., Jurcic, K., Schneider, C., Karr, D., Fisher, G.L., Fridgen, T.D., Diego-Taboada, A., Georghiou, P.E., Mackenzie, G., Banoub, J., 2020. Demystifying and unravelling the molecular structure of the biopolymer sporopollenin. Rapid Commun. Mass Spectrom. 34, e8740. https://doi.org/10.1002/rcm.8740.

Mundargi, R.C., Potroz, M.G., Park, J.H., Seo, J., Tan, E.L., Lee, J.H., Cho, N.J., 2016. Eco-friendly streamlined process for sporopollenin exine capsule extraction. Sci. Rep. 6, 19960. https://doi.org/10.1038/srep19960.

Nabil, B., Ouaabou, R., Ouhammou, M., Saadouni, L., Mahrouz, M., 2019. Impact of particle size on functional, physicochemical properties and antioxidant activity of cladode powder (Opuntia ficus-indica). J. Food Sc. Technol. https://doi.org/10.1007/s13197019-04127-4.

Orhan, I., Özçelik, B., Aslan, S., Kartal, M., Karaoglu, T., Sener, B., Terzioglu, S., Choudhary, M.I., 2007. . Phytochem. Rev. 6, 189-196. https://doi.org/10.1007/s11101006-9053-x.

Paquot, C., Hautfenne, A., 1971. Peroxide Value, in: Standards Methods for the Analysis of Oils, Fats and Derivatives. IUPAC.
Prior, R.L., Wu, X., Schaich, K., 2005. Standardized methods for the determination of antioxidant capacity and phenolics in foods and dietary supplements. J. Agric. Food Chem. 53, 4290-4302. https://doi.org/10.1021/jf0502698.

Rozema, J., Broekman, R.A., Blokker, P., Meijkamp, B.B., de Bakker, N., van de Staaij, J., van Beem, A., Ariese, F., Kars, S.M., 2001. UV-B absorbance and UV-B absorbing compounds (para-coumaric acid) in pollen and sporopollenin: the perspective to track historic UV-B levels. J. Photochem. Photobiol. B, Biol. 62, 108-117. https://doi.org/ 10.1016/S1011-1344(01)00155-5.

Rozema, J., Noordijk, J., Broekman, R.A., van Beem, A., Meijkamp, B.M., de Bakker, N.V.J., van de Staaij, J.W.M., Stroetenga, M., Bohncke, S.J.P., Konert, M., Kars, S., Peat, H., Smith, R.I.L., Convey, P., 2001. (Poly)phenolic compounds in pollen and spores of Antarctic plants as indicators of solar UV-B - A new proxy for the reconstruction of past solar UV-B?. Plant Ecol. 154, 9-26.

Sahiner, M., Sahiner, N., Sagbas, S., Fullerton, M.L., Blake, D.A., 2018. Fabrication of biodegradable poly(naringin) particles with antioxidant activity and low toxicity. ACS Omega 3 (12), 17359-17367. https://doi.org/10.1021/acsomega.8b02292.

Schulze Osthoff, K., Wiermann, R., 1987. Phenols as integrated compounds of sporopollenin from Pinus pollen. J. Plant Physiol. 131, 5-15. https://doi.org/10.1016/S01761617(87)80262-6.

Scott, G., 1997. Antioxidants in Science, Technology, Medicine and Nutrition. Albion Publishing Ltd., Chichester.

Serpen, A., Capuano, E., Fogliano, V., Gökmen, V., 2007. A new procedure to measure the antioxidant activity of insoluble food components. J. Agr. Food Chem. 55, 7676-7681. https://doi.org/10.1021/jf071291z.

Sharma, O.P., Bhat, T.K., 2009. DPPH antioxidant assay revisited. Food Chem. 113, 1202-1205. https://doi.org/10.1016/j.foodchem.2008.08.008.

Shaw, G., 1971. The chemistry of sporopollenin. In: Brooks, J, Grant, P.R., Muir, M. (Eds.), Sporopollenin,. Academic Press, London \& New York, pp. 305-348.

Smirnova, A.V., Timofeyev, K.N., Breygina, M.A., Matveyeva, N.P., Yermakov, I.P., 2012. Antioxidant properties of the pollen exine polymer matrix. Biophysics 57, 174-178. https://doi.org/10.1134/S0006350912020224.

Soualeh, N., Stiévenard, A., Bouayed, J., Soulimani, R., 2018. Powders with small microparticle size from Hedera helix and Scrophularia nodosa exhibited high preventive antioxidant activity against $\mathrm{H}_{2} \mathrm{O}_{2}$-induced oxidative stress in mouse primary spleen cells. Int. J. Vitam. Nutr. Res. 88, 208-218. https://doi.org/10.1024/0300-9831/ a000526.

Thomasson, M.J., Baldwin, D.J., Diego-Taboada, A., Atkin, S.L., Mackenzie, G., Wadhawan, J.D., 2010. Electrochemistry and charge transport in sporopollenin particle arrays. Electrochem. commun. 12, 1428-1431. https://doi.org/10.1016/j.elecom.2010. 07.038.

Torkamani, A.E., Syahariza, Z.A., Norziah, M.H., Mahmood, W.A.K., Juliano, P., 2018. Production and characterization of gelatin spherical particles formed via Electrospraying and encapsulated with polyphenolic antioxidants from Momordica charantia. Food Bioprocess Technol. 11, 1943-1954. https://doi.org/10.1007/s11947-018-2153-y.

Vinter, H., 1995. Production of high quality Fish oils. In: Hamilton, R.J., Rice, R.D. (Eds.), Fish Oil: Technology, Nutrition and Marketing. Barnes and Associates, High Wycombe, pp. 27-33.

Wehling, K., Niester, C., Boon, J.J., Willemse, M.T., Wiermann, R., 1989. P-coumaric acid - a monomer in the Sporopollenin skeleton. Planta 179, 376-380. https://doi.org/10. 1007/BF00202338.

Zaiter, A., Becker, L., Karam, M.C., Dicko, A., 2016. Effect of particle size on antioxidant activity and catechin content of green tea powders. J. Food Sci. Technol. 53, 2025-2032. https://doi.org/10.1007/s13197-016-2201-4.

Zetzsche, F., Huggler, K., 1928. Untersuchungen über die Membran des Sporen und Pollen I. 1. Lycopodium clavatum L. Liebigs Ann. Chem 461, 89-108. https://doi.org/10. 1002/jlac.19284610105.

Zetzsche, F., Kalin, O., 1931. Untersuchungen über die Membran der Sporen und Pollen V. 4. Zur Autoxydation der Sporopollenine. Helv. Chim. Acta 14, 517-519. https://doi. org/10.1002/hlca.19310140151. 